\title{
Population pharmacokinetics of rhTNFR-Fc in healthy Chinese volunteers and in Chinese patients with Ankylosing spondylitis
}

Yi FANG ${ }^{1,2}$, Lu-jin $\mathrm{LI}^{3}$, Rui WANG ${ }^{4}$, Feng HUANG ${ }^{4}$, Hai-feng SONG ${ }^{5}$, Zhong-ming TANG ${ }^{5}$, Yu-zhen $\mathrm{LI}^{2}$, Hua-shi GUAN ${ }^{1, *}$, Qing-shan $\mathrm{ZHENG}^{3, *}$

${ }^{1}$ Marine Drug and Food Institute, Ocean University of China, Qingdao 266003, China; ${ }^{2}$ Peking University People's Hospital, Beijing 100044, China; ${ }^{3}$ Shanghai University of Chinese Medicine, Shanghai 201203, China; ${ }^{4}$ Department of Clinical Pharmacology, Chinese PLA General Hospital, Beijing, 100853, China; ${ }^{5}$ Beijing Institute of Radiation Medicine, Academy of Military Medical Science, Beijing 100850, China

Aim: To investigate the population pharmacokinetics of recombinant human tumor necrosis factor receptor-Fc fusion protein (rhTNFRFc) administered via subcutaneous (SC) injection in healthy Chinese volunteers and in Chinese patients with ankylosing spondylitis (AS).

Methods: Thirty-two healthy volunteers were randomly assigned to receive a single SC injection of $12.5,25,37.5$, or 50 mg of rhTNFRFc. Twenty male patients with moderate AS were randomly assigned to receive seven consecutive SC injections of rhTNFR-Fc at either $25 \mathrm{mg}$ twice a week (BIW) or $50 \mathrm{mg}$ once a week (QW). Population pharmacokinetic (PK) analysis was applied to obtain PK parameters of rhTNFR-Fc by the NONMEM method.

Results: The data were best described by a one-compartment model with lag time. We found that gender had a significant effect on the apparent clearance $(\mathrm{CL} / \mathrm{F})$, with the male $\mathrm{CL} / \mathrm{F}$ ratio being only 0.665 times the female ratio; the absorption coefficient $(\mathrm{F})$ of multiple dosages of rhTNFR-Fc was only 0.674 times that of a single dosage. The outcome parameters were $\mathrm{CL} / \mathrm{F}$ (female: $0.168 \mathrm{~L} / \mathrm{h}$, male: $0.110 \mathrm{~L} / \mathrm{h}$ ), the apparent volume of distribution (Vd/F: $15.5 \mathrm{~L}$ ), the absorption rate constant (Ka) (single dosage: $0.0605 \mathrm{~h}^{-1}$, multiple dosage: $0.0408 \mathrm{~h}^{-1}$ ), and the lag time ( $\mathrm{T}_{\text {lag: }}: 1.03 \mathrm{~h}$ ). The inter-individual variability in the $\mathrm{CL} / \mathrm{F}, \mathrm{Vd} / \mathrm{F}, \mathrm{Ka}$, and $\mathrm{T}_{\text {lag }}$ were $33.3 \%, 42.7 \%$, $55.6 \%$, and $81.8 \%$, respectively.

Conclusion: Chinese females have a higher CL/F than Chinese males, and multiple dosings can significantly decrease the absorption of rhTNFR-Fc (SC). The population PK parameters of rhTNFR-Fc in healthy Chinese volunteers and patients with AS were similar to those reported for subjects in published American studies.

Keywords: tumor necrosis factor receptor; fusion protein; ankylosing spondylitis; population pharmacokinetics

Acta Pharmacologica Sinica (2010) 31: 1500-1507; doi: 10.1038/aps.2010.113; published online 18 Oct 2010

\section{Introduction}

Ankylosing spondylitis (AS) is a chronic inflammatory disease that affects the spinal and sacroiliac joints. This disease affects more than 3 million people in China, most of whom are young adults (16-40 years old $)^{[1]}$. Many patients have localized new bone formation, ligamentous ossification, and progressive deformities that, without treatment, often lead to disability. A recent Norwegian study showed that the cessation of physical work in patients with AS occurred at an average disease

\footnotetext{
* To whom correspondence should be addressed.

E-mail zhengqscn@21cn.com (Qing-shan ZHENG); hsguan@ouc.edu.cn (Hua-shi GUAN)

Received 2010-04-12 Accepted 2010-07-07
}

duration of 15.6 years ${ }^{[2]}$. Currently, there is no cure for AS, but available therapies, such as individualized physical therapy and nonsteroidal anti-inflammatory drugs (NSAIDs), target symptoms and pain ${ }^{[3]}$.

Tumor necrosis factor-alpha (TNF- $\mathrm{a})$ is a proinflammatory cytokine implicated in a variety of inflammatory diseases, including $\mathrm{AS}^{[4]}$. Produced primarily by macrophages and activated lymphocytes, TNF-a upregulates the expression of multiple inflammatory factors, such as interleukin 1 (IL-1), IL-6, prostaglandins, and matrix metalloproteases ${ }^{[5]}$. Transgenic mice expressing truncated Peromyscus leucopus TNF-a developed inflammatory axial arthritis that morphologically resembled $A S^{[6]}$. The levels of TNF-a in serum from patients with AS are much higher than those in healthy subjects, although 
no significant correlation between TNF-a level and the severity of AS has been observed ${ }^{[7]}$. Furthermore, elevated levels of TNF- $\alpha$ mRNA and protein were found in biopsy specimens of the sacroiliac joints of patients with $\mathrm{AS}^{[8,9]}$.

TNF-a blockade therapy has proved to be an effective treatment strategy for AS. Etanercept (the first rhTNFR-Fc product) is a dimeric fusion protein consisting of the extracellular ligand-binding portion of the human TNF receptor (TNFR) linked to the Fc portion of human IgG1 (rhTNFR-Fc) ${ }^{[10]}$. The rhTNFR-Fc protein binds to TNF-a and thereby blocks the interaction between TNF- $\alpha$ and its cell surface receptors. Multiple randomized controlled trials demonstrated that etanercept treatment was able to effectively control symptoms, to prevent both clinical and radiographic progression of disease, and to improve the quality of life of patients with $A S^{[11-15]}$. Etanercept has been approved in the United States and Europe for the treatment of AS, and clinical investigations of etanercept in the treatment of AS and rheumatoid arthritis are also underway in China. Although the pharmacokinetics (PK) of rhTNFR-Fc in the American population have been well documented $^{[16,17]}$, it is not clear whether ethnic differences affect the PK characteristics of rhTNFR-Fc in the Chinese population. In this study, we investigated the population PK of rhTNFR-Fc in healthy Chinese volunteers and in Chinese patients with moderate AS.

\section{Materials and methods Drugs and instruments}

The rhTNFR-Fc (batch numbers 20041101-1 and 20041101-2) was provided by Celgen Bio-Pharmaceutical Co Ltd (Shanghai, China) as a lyophilized powder and stored at $0-4{ }^{\circ} \mathrm{C}$ in the dark until use. Before each administration, the lyophilized powder was dissolved in $1 \mathrm{~mL}$ of sterile water and injected immediately.

Plasma samples were prepared using a Heraeus Biofuge 22R refrigerated high-speed centrifuge. Enzyme-linked immunosorbent assay (ELISA) readings were performed with a PolarStar Galaxy fluorescent plate reader (BMG Labtech, Durham, NC, USA).

\section{Patients and healthy subjects}

This study was approved by the Ethics Committee of the General Hospital of the People's Liberation Army (Beijing, China). Written informed consent was obtained in accordance with the Good Clinical Practice guidelines published by the State Food and Drug Administration of China in 2003. Thirty-two healthy Chinese volunteers (16 males, 16 females, aged 25 to 35 years, whose body mass indices ranged from 19 to 24 ) were enrolled to receive a single dose of rhTNFR-Fc. The health status of all healthy subjects was confirmed by routine physical examination and standard laboratory tests. The participants had no history of immune disease, liver or kidney disease, hemorrhagic disease, heart or cardiovascular disease, nervous system disease, hematological disease, chronic infection or other serious infections, organ transplantation, drug or food allergy, drug or alcohol abuse, or tuberculosis (TB) or contact with high-risk patients with TB. No female volunteers were pregnant or nursing at the time of the study.

Twenty male Chinese patients with moderate and active AS (20 to 31 years old and body mass indices from 19 to 24 ) were enrolled to receive multiple injections of rhTNFR-Fc. Inclusion was dependent on a diagnosis of AS based on the New York criteria published in 1968. Exclusion criteria included complete tetanus; serious disease of the heart, liver, kidney, or endocrine system; disseminated sclerosis; prior anti-TNF therapy; infection (acute or chronic); HIV; HbsAg-positive status; and a history of tuberculosis or a positive tuberculosis skin test. The patients continued to receive NSAIDs during the study if they had been taking them for at least 4 months prior to enrollment. Physical therapy and glucocorticoid administration were also allowed during the study.

\section{Study design}

Healthy volunteers received escalating doses of rhTNFR-Fc (eight subjects at each dose level, only one dose per patient): $12.5,25,37.5$, and $50 \mathrm{mg}$. The study of the next largest dose was initiated only after the safety of the previous dose had been established. Each healthy volunteer received a single subcutaneous injection of rhTNFR-Fc in the abdomen at 8 am (before breakfast).

Patients with AS were assigned (10 patients per group) to receive seven consecutive subcutaneous injections of rhTNFRFc in the abdomen at doses of either $25 \mathrm{mg}$ twice a week (BIW) or $50 \mathrm{mg}$ once a week $(\mathrm{QW})$. All injections were administered at 8 am (before breakfast). However, one patient in the $50 \mathrm{mg}$ QW group missed a dose and was excluded from the study.

Light, balanced diets (2000-3000 calories/day) were provided to all subjects, although absolute fasting at night (after $10 \mathrm{pm}$ ) was required. Caffeine, tea, tobacco, and alcohol were also prohibited in this study.

Serial blood samples were collected in anticoagulant tubes containing EDTA at specified times during the study $(2,4,12$, $24,36,48,60,72,84,96,120,144,168,216,264,312,384$, and $480 \mathrm{~h}$ after the first dose). Plasma samples were isolated from fresh blood by centrifugation and stored at $-20^{\circ} \mathrm{C}$.

\section{Determination of Plasma Levels of rhTNFR-Fc}

Plasma levels of rhTNFR-Fc were determined by ELISA using the Quantikine ${ }^{\circledR}$ human sTNF RII immunoassay kit (R \& D Systems Co, Minneapolis, MN, USA). This assay was validated for the determination of rhTNFR-Fc fusion protein in human plasma (data not shown). A standard curve was established using rhTNFR-Fc fusion protein as a reference. Linearity and reproducibility were achieved in the range of 48.8 to $3125 \mathrm{pg} / \mathrm{mL}$.

\section{Basic model selection}

Based on the rhTNFR-Fc concentration-versus-time curves from the healthy subjects and AS patients and related reports ${ }^{[16,17]}$, the one- and two-compartment models with firstorder absorption and elimination were chosen as basic models. The data were fit without introducing any covariates. The 
objective function value (OFV), data fitness, and parameter rationality were comprehensively compared to choose the best model.

\section{Building models of random effects}

The random effects of population pharmacokinetics included inter-individual variability and residual error. Exponential models were used to account for inter-individual variability. Therefore, the distribution of the pharmacokinetic parameters was defined as:

$$
P_{\mathrm{i}}=P_{\mathrm{TV}} \cdot \operatorname{Exp}\left(\eta_{\mathrm{i}}\right),
$$

where $P_{\mathrm{i}}$ is the estimated parameter value of the individual $i, P_{\mathrm{TV}}$ is the typical population value of the parameter, and $\eta_{\mathrm{i}}$ represents the inter-individual random effects for individual $\mathrm{i}$ and is assumed to be normally distributed: $\eta_{\mathrm{i}} \sim \mathrm{N}\left(0, \omega^{2}\right)$, with inter-individual variance-covariance matrix $\Omega$.

$$
C_{\text {obs }}=C_{\text {pred }}{ }^{*}\left(1+\varepsilon_{1}\right)+\varepsilon_{2}
$$

where $C_{\text {obs }}$ and $C_{\text {pred }}$ are observed and predicted concentrations, respectively. The parameter $\varepsilon$ represents the residual error of the model, which is a normally distributed random variable with a mean of zero and variance $\sigma^{2}$, and $\varepsilon_{1}$ and $\varepsilon_{2}$ denote proportional and additive residual error, respectively.

\section{Building the covariate model}

Once the basic model was selected, the relationship between the covariates and intra-individual random effects ( $\eta$ ) were assessed with a scatter plot. During modeling, the covariates, in the form of additive or exponential models, were introduced into the basic model to perform the population pharmacokinetic analysis.

\section{Building the full regression model}

Stepwise regression was used to establish the full regression model. After one covariate was introduced into the model and the OFV decreased by more than $6.64\left(X_{0.01,1}^{2}\right)$, the model was significantly affected $(P<0.01)$, indicating that this covariate should be retained. Continuing this procedure, all significant covariates were found and included to establish the full regression model.

Reverse exclusion to establish the final model

The added covariates were then excluded one-by-one from the full regression model. The more stringent statistical criteria were used to test the necessity of each covariate included in this model. If the OFV change was more than $10.83\left(\chi_{0.001,1}^{2}\right)$, the effect was deemed significant $(P<0.001)$ and was kept in the model. However, if the OFV change was less than 10.83, this covariate was removed from the model. The final model was obtained after the reverse removal of covariates from the full regression model.

\section{Validation of the final model}

In our study, the bootstrap method was used to validate the model. The individual data from identical samples were collected from the total set of individual samples to obtain a new data file, which was then analyzed by NOMMEM. The median values and $95 \%$ confidence intervals for the parameter estimates obtained from 1000 bootstrap replications with successful runs (both the estimation and covariance steps successfully converged) were compared with those obtained from the original data set.

\section{Analysis software}

NOMMEM, version V level 11.1 (GloboMax, LLC, USA), and DAS, version 3.0 (Professional Committee of Pharmacometrics of China, China), were used for data analysis.

\section{Results}

\section{Demographic information about the subjects}

In our study, 1187 blood samples from a total of 51 volunteers were collected. The demographic factors, including gender, age, height, and weight (WT), as well as laboratory indicators, including alanine aminotransferase (ALT), aspartate aminotransferase (AST), blood urea nitrogen (BUN), and serum creatinine $(\mathrm{Cr})$, were recorded as the covariates for the population pharmacokinetic studies (Table 1). In addition, we also evaluated the effects of drug administration times (either single or multiple dosings) and the health status of the subjects (either healthy or with AS) on the pharmacokinetic parameters.

\section{Mean concentration-time profiles of rhTNFR-Fc in serum}

A total of 51 volunteers received subcutaneous injections of rhTNFR-Fc. Healthy subjects received single injections of 12.5, $25,37.5$, or $50 \mathrm{mg}$, whereas patients with AS patients received

\begin{tabular}{|c|c|c|c|c|c|c|}
\hline Characteristic & $\begin{array}{c}\text { Single dose } \\
12.5 \mathrm{mg}\end{array}$ & $\begin{array}{l}\text { Single dose } \\
25 \mathrm{mg}\end{array}$ & $\begin{array}{l}\text { Single dose } \\
37.5 \mathrm{mg}\end{array}$ & $\begin{array}{l}\text { Single dose } \\
50 \mathrm{mg}\end{array}$ & $\begin{array}{l}\text { Multiple dose } \\
25 \mathrm{mg}\end{array}$ & $\begin{array}{l}\text { Multiple dose } \\
50 \mathrm{mg}\end{array}$ \\
\hline Gender (male/female) & $4 / 4$ & $4 / 4$ & $4 / 4$ & $4 / 4$ & $10 / 0$ & $9 / 0$ \\
\hline Age (years) & $30.13 \pm 3.76$ & $32.13 \pm 3.48$ & $31.38 \pm 2.45$ & $30.75 \pm 2.76$ & $24.50 \pm 4.06$ & $24.89 \pm 3.55$ \\
\hline Height (cm) & $166.00 \pm 8.60$ & $163.25 \pm 8.71$ & $164.50 \pm 6.57$ & $165.25 \pm 4.43$ & $171.80 \pm 4.52$ & $173.22 \pm 5.26$ \\
\hline Weight (kg) & $60.25 \pm 7.27$ & $60.00 \pm 5.35$ & $60.88 \pm 6.64$ & $60.38 \pm 6.59$ & $64.40 \pm 7.12$ & $66.11 \pm 7.65$ \\
\hline $\mathrm{BUN}(\mu \mathrm{mol} / \mathrm{L})$ & $4.43 \pm 0.90$ & $5.28 \pm 1.86$ & $4.52 \pm 1.01$ & $4.40 \pm 1.10$ & $4.62 \pm 1.15$ & $5.21 \pm 1.29$ \\
\hline $\mathrm{Cr}(\mu \mathrm{mol} / \mathrm{L})$ & $52.95 \pm 9.12$ & $52.25 \pm 11.89$ & $53.40 \pm 9.96$ & $51.48 \pm 9.20$ & $61.57 \pm 9.43$ & $64.72 \pm 9.40$ \\
\hline Aspartate transaminase $(\mathrm{U} / \mathrm{L})$ & $11.56 \pm 3.59$ & $13.64 \pm 5.06$ & $18.19 \pm 6.24$ & $24.36 \pm 15.57$ & $17.71 \pm 9.78$ & $20.02 \pm 15.78$ \\
\hline Alanine transaminase $(\mathrm{U} / \mathrm{L})$ & $18.46 \pm 2.32$ & $16.09 \pm 1.97$ & $16.25 \pm 2.30$ & $22.54 \pm 7.51$ & $19.17 \pm 4.04$ & $18.16 \pm 4.80$ \\
\hline
\end{tabular}

Table 1. Demographic data of subjects included in population analysis. 

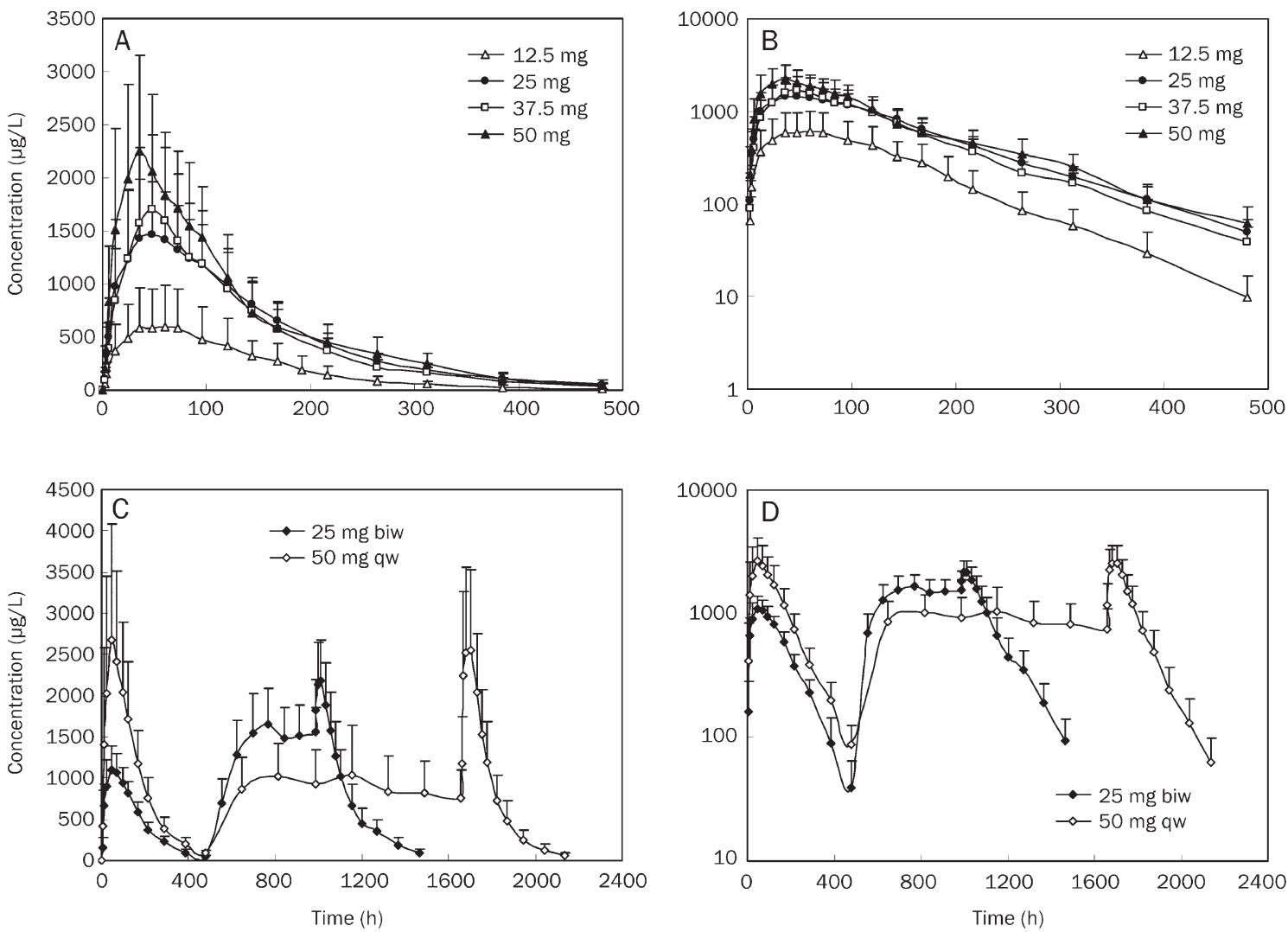

Figure 1. The mean concentration-time curves for healthy volunteers after received a single subcutaneous injection of rhTNFR-Fc (Fc $12.5,25,37.5$, $50 \mathrm{mg}, n=8)(\mathrm{A})$ and its log concentration-time graph (B). Concentration-time curves for patients with ankylosing spondylitis after received multiple subcutaneous injections of rhTNFR-Fc (25 mg BIW, $n=10$ and $50 \mathrm{mg} \mathrm{QW}, n=9)$ (C) and its log concentration-time graph (D).

either $25 \mathrm{mg}$ BIW or $50 \mathrm{mg}$ QW. The mean concentration-time profiles for the different dose groups are shown in Figure 1. Based on the profiles of the curves and on related literature reports, one- and two-compartment models were initially chosen as the basic models for this study.

\section{Basic model}

We compared the OFV of the one- and two-compartment models with a time lag $\left(\mathrm{T}_{\text {lag }}\right)$ and found that the OFV value of the one-compartment model was decreased by 893.982 $(P<0.001)$ compared with the two-compartment model. Otherwise, the fit of the one-compartment model indicated that the population predictive value (PRED), individual predictive value (IPRED), and observation value (OBS) had better correlations (Figure 2). The trend line was close to the diagonal, and the weighted residuals (WRES) value was more evenly distributed on both sides of the axis, with most of the data located between \pm 4 , suggesting that the one-compartment model was preferable. Therefore, the one-compartment model was chosen as the basic model for the next population pharmacokinetic study.

\section{Full regression model}

The demographic factors, laboratory indicators, single or multiple drug dosages, and state of the subjects were considered as covariates. The effects on the OFV were investigated after each covariate was added into the pharmacokinetic basic model. The processes of stepwise establishment of the regression model and reverse exclusion are shown in Table 2. The modeling process showed that single or multiple drug dosages $(\mathrm{M})$ had a significant effect on the $\mathrm{F}$ value (absorption coefficient). In addition, gender had a significant effect on the $\mathrm{CL} / \mathrm{F}$. The reverse removal process showed that the effects of $\mathrm{M}$ on the $\mathrm{F}$ value and the effects of gender on the $\mathrm{CL} / \mathrm{F}$ value were significant. Therefore, these covariates were retained in the final model.

Table 2. Population pharmacokinetic modeling process.

\begin{tabular}{llcc}
\hline № & \multicolumn{1}{c}{ Model description } & OFV & OFV \\
\hline \multicolumn{2}{l}{ Model forward inclusion } & & \\
1 & Basic model (one-compartment model) & 13837.487 & \\
2 & Add $\mathrm{M}$ to $\mathrm{F}$ based on model 1 $\left(\mathrm{F}^{*} \theta^{\wedge} \mathrm{M}\right)$ & 13481.093 & -356.394 \\
3 & Add gender to $\mathrm{CL} / \mathrm{F}$ based on model 2 & 13375.985 & -105.108 \\
& $\left(\mathrm{CL} / \mathrm{F}^{\star} \theta^{\wedge}\right.$ Gender) & & \\
& & & \\
Model backward elimination & 13481.093 & 105.108 \\
4 & Remove gender from $\mathrm{CL} / \mathrm{F}$ in model 3 & 13730.810 & 354.825 \\
5 & Remove $\mathrm{M}$ from $\mathrm{F}$ in model 3 &
\end{tabular}



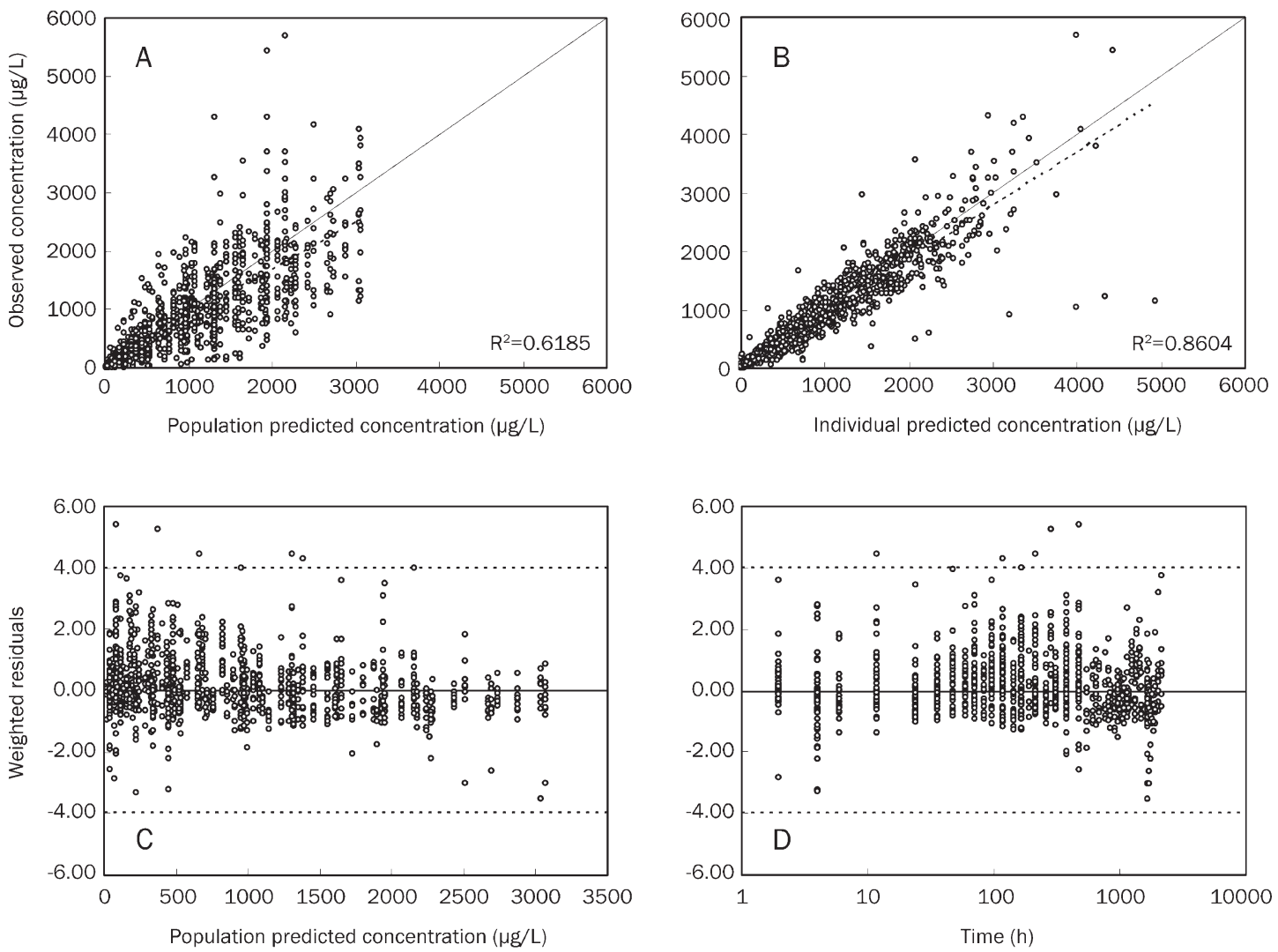

Figure 2. Basic model evaluation plots. (A) Population predicted concentration vs. Observed concentration. (B) Individual predicted concentration vs. observed concentration. (C) Weighted residuals vs population predicted concentration. (D) Sample time vs. weighted residuals. The solid line in (A) and (B) represent the line of identity, and those in $(C)$ and $(D)$ represent the line $y=0$. The dotted lines in $(A)$ and $(B)$ are linear regression line, and those in $(C)$ and $(D)$ are the limit lines $y= \pm 4$.

\section{Final model and the estimation of parameters}

The final model for rhTNFR-Fc population pharmacokinetics was as follows:

$$
\begin{aligned}
& \mathrm{F}_{\mathrm{i}}=\mathrm{F}^{*} 0.674^{\mathrm{M}} \\
& (\mathrm{CL} / \mathrm{F})_{\mathrm{i}}=0.168^{*} 0.655^{\text {Gender } *} \operatorname{Exp}\left(\mathrm{\eta}_{\mathrm{CL} / \mathrm{Fi}}\right) \mathrm{L} / \mathrm{h}^{-1}
\end{aligned}
$$

where $\mathrm{M}$ was 0 for a single dosage and 1 for multiple dosages, and gender was 0 for a female and 1 for a male. The correction factor of $M$ versus $F$ was 0.674 , which indicated that the absorption of multiple dosages of drug divided by the absorption of a single dosage of drug was 0.674 . The correction factor of gender on $\mathrm{CL} / \mathrm{F}$ was 0.665 , which indicated that the clearance rate in males was only 0.665 of that in females.

The population pharmacokinetic parameters and the estimate of parameter variance in the final model are shown in Table 3. The standard error of all the parameters was less than $30 \%$, except for the standard error of inter-individual variability with $\mathrm{T}_{\text {lag }}$ and the additive error, both of which exceeded $30 \%$.

The fit of the data to the final model demonstrated that PRED and IPRED could predict OBS better than the basic model and that the squares of their correlation coefficient values were all significantly increased. The scatter plots of WRES versus PRED and time were evenly distributed around a straight line through 0 and were located within \pm 4 of the center (Figure 3). Our data showed, through the correction of $\mathrm{M}$ on $\mathrm{F}$ and gender on $\mathrm{CL} / \mathrm{F}$, that both the accuracy and precision predictions were improved significantly. Figure 4 shows the distribution of the observed concentrations against the population predicted concentrations of different administration groups.

\section{Model validation}

Analysis of each of the 1000 bootstrap samples resulted in 997 samples that successfully converged. The median values of the parameters after 997 repetitions of the Bootstrap estimation were consistent with the parameter estimates of the original samples (Table 3), and the $95 \%$ confidence interval was within reasonable limits. Furthermore, the 95\% confidence interval of the $\mathrm{M}$ and gender correction factors did not include 1 , suggesting that the final population pharmacokinetic model was stable.

\section{Discussion}

Although the pathogenesis of AS is not fully understood, it is clear that TNF-a plays a key role in the inflammatory processes in patients with AS. Consequently, TNF-a has emerged as an important therapeutic target for AS, and TNF-a blockade therapy has greatly improved the clinical management of $\mathrm{AS}^{[4]}$. 

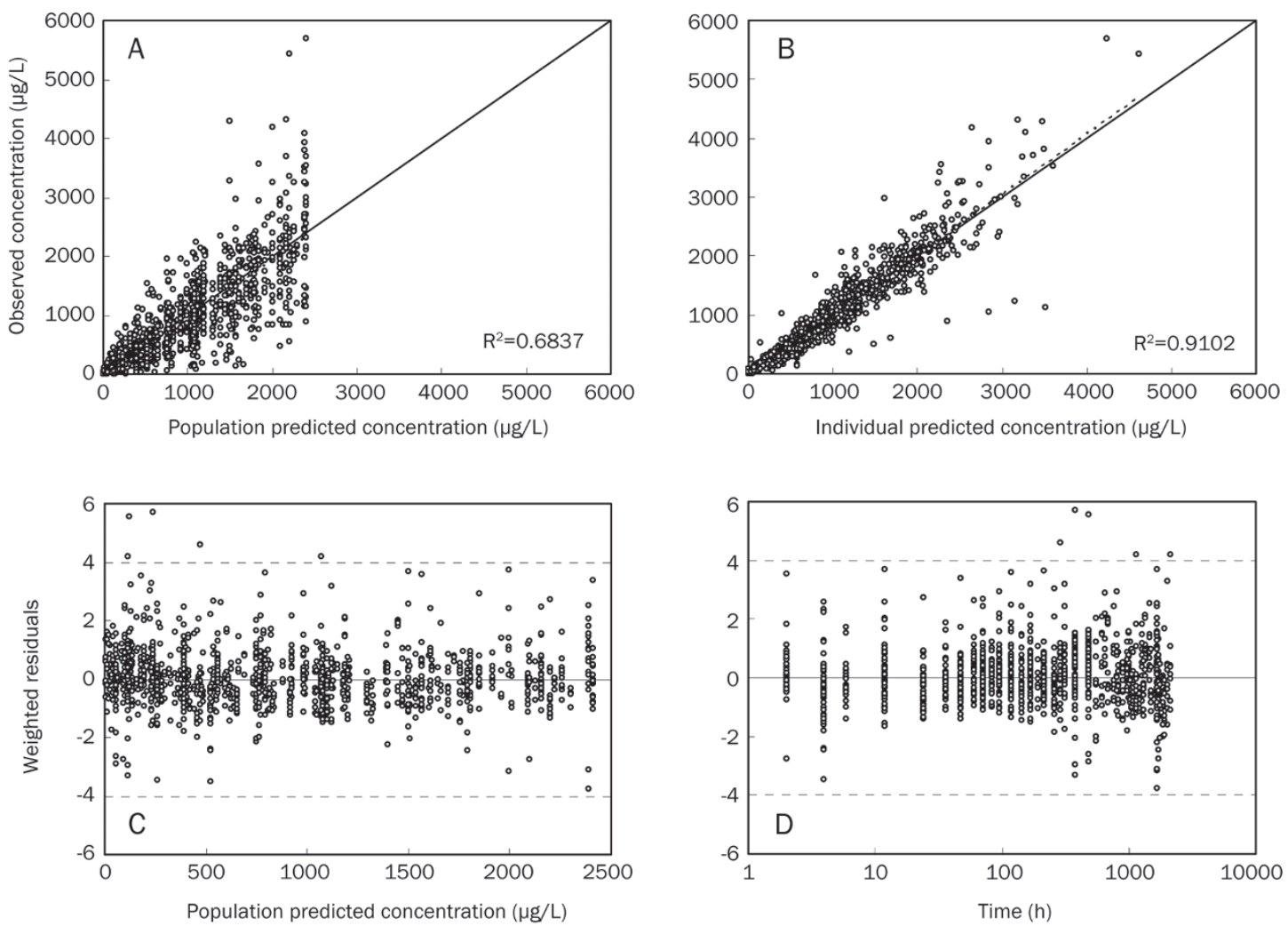

Figure 3. Final model evaluation plots. (A) Population predicted concentration vs observed concentration. (B) Individual predicted concentration vs observed concentration. (C) Weighted residuals vs population predicted concentration. (D) Sample time vs weighted residuals. The solid line in (A) and (B) represent the line of identity, and those in $(C)$ and $(D)$ represent the line $y=0$. The dotted lines in $(A)$ and $(B)$ are linear regression line, and those in $(C)$ and $(D)$ are the limit lines $y= \pm 4$.

Table 3. Parameter estimates of the final model.

\begin{tabular}{lcc}
\hline \multicolumn{1}{c}{ Parameter } & $\begin{array}{c}\text { Estimates of } \\
\text { the model } \\
\text { parameters (SE\%) }\end{array}$ & $\begin{array}{c}\text { Bootstrapped median } \\
(95 \% \mathrm{Cl})\end{array}$ \\
\hline Pharmacokinetic parameter & \\
$\mathrm{CL} / \mathrm{F}, \mathrm{L} / \mathrm{h}$ & $0.168(7.1)$ & $0.166(0.053-0.194)$ \\
$\mathrm{V} / \mathrm{F}, \mathrm{L}$ & $15.5(6.3)$ & $15.4(5.25-17.5)$ \\
$\mathrm{Ka}, 1 / \mathrm{h}$ & $0.0605(9.9)$ & $0.0601(0.0477-0.0711)$ \\
$\mathrm{T}_{\text {lag, }} \mathrm{h}$ & $1.03(14.0)$ & $1.05(0.71-1.31)$ \\
$\theta_{\mathrm{M}}$ for $\mathrm{F}$ & $0.674(6.8)$ & $0.673(0.57-0.75)$ \\
$\theta_{\text {Gender }}$ for CL/F & $0.655(6.8)$ & $0.666(0.57-0.84)$ \\
& & \\
Interindividual variability & & \\
$\mathrm{CL} / \mathrm{F}, \%$ & $33.3(23.5)$ & $32.9(25.1-62.8)$ \\
$\mathrm{V} / \mathrm{F}, \%$ & $42.7(27.3)$ & $41.8(30.0-63.0)$ \\
Ka, \% & $55.6(19.6)$ & $55.2(22.1-67.2)$ \\
$\mathrm{T}_{\text {lag }}, \%$ & $81.8(74.4)$ & $74.1(28.5-164.0)$ \\
& & \\
Residual variability & & $20.0(6.9-25.6)$ \\
Proportional error, \% & $20.3(29.6)$ & $12.6(2.5-24.5)$ \\
Additive error, $\mu \mathrm{g} / \mathrm{L}$ & $12.6(62.8)$ & \\
\hline
\end{tabular}

Current TNF-a blocking agents fall into two categories: antiTNF-a monoclonal antibodies and rhTNFR-Fc fusion proteins.
Etanercept (Enbrel ${ }^{\circledR}$, Amgen \& Wyeth) is the first rhTNFR-Fc approved for the treatment of AS. The pharmacokinetics of rhTNFR-Fc in the American population have been well documented $^{[16,17]}$. However, little is known about the effect of ethnic differences on the PK of rhTNFR-Fc, particularly in the Chinese population.

This study establishes rhTNFR-Fc population pharmacokinetic models in both healthy Chinese subjects and in Chinese patients with AS and provides a basis for further clinical studies. During the process of selecting the structure model, we compared the fits of the one- and two-compartment models with first-order absorption and elimination. Our data showed that the one-compartment model more accurately described the pharmacokinetic features of rhTNFR-Fc. Therefore, a onecompartment model with time lag was finally selected as the basic model, which was consistent with previous reports of rhTNFR-Fc pharmacokinetics ${ }^{[16,17]}$.

During the process of covariate selection, we compared the effects of height, weight, age, gender, AST, ALT, BUN, $\mathrm{Cr}$, dose number, and the health status of the subjects on the pharmacokinetic parameters CL/F, V/F, and Ka. Our data showed that gender had a significant effect on $\mathrm{CL} / \mathrm{F}$, with the male CL/F ratio only 0.665 times the female ratio. Gender differences in the PK of rhTNFR-Fc have also been reported by other investigators, but their results were discordant with 

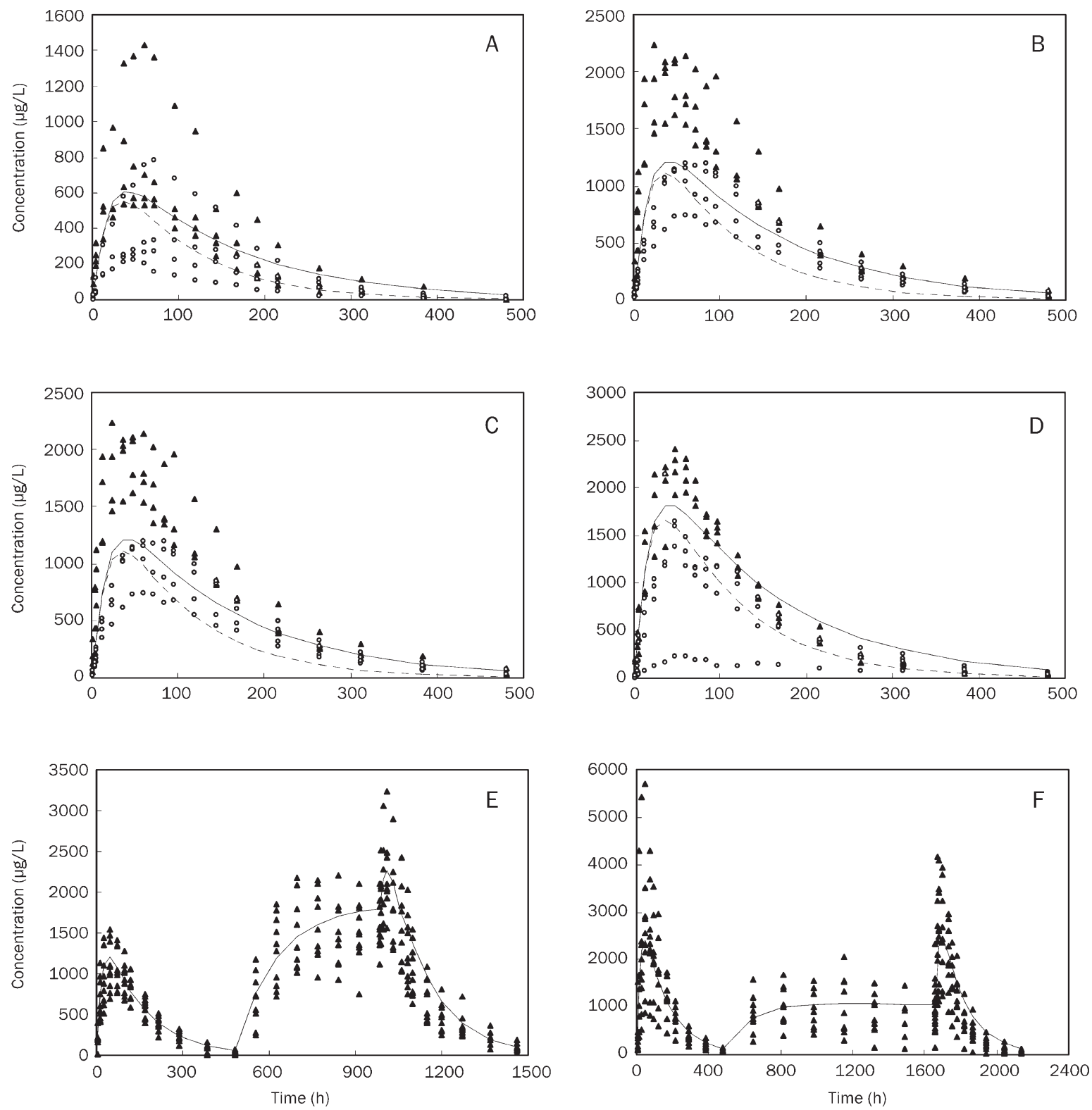

Figure 4. Population predicted concentration vs. observed concentration. (A) single dose of $12.5 \mathrm{mg}$; (B) single dose of $25 \mathrm{mg}$; (C) single dose of 37.7 mg; (D) single dose of $50 \mathrm{mg}$; (E) single and multiple doses of $25 \mathrm{mg}$, BIW; (F) single and multiple doses of $50 \mathrm{mg}$, QW. The hollow point represents the observed concentration in the female subjects, the solid point represents the observed concentration in the male subjects, the dotted line represents the population predictive concentration in the women subjects and the solid line represents the population predictive value in the male subjects.

ours. For example, Lee et al ${ }^{[18]}$ reported that the CL/F of males is higher than that of females, though the difference did not reach statistical significance. Yim et al also reported similar results ${ }^{[19]}$. The biological basis for these observed gender differences is currently unknown, and whether the dose of rhTNFR-Fc should be adjusted according to gender should be investigated in larger trials. In addition, we found that the number of dosings affects both the CL/F and V/F. After the introduction of the factor of dose number into $\mathrm{F}$, the OFV was significantly decreased, and the effects of the dose number on CL/F and V/F disappeared, suggesting that dose number could significantly affect drug absorption without affecting the clearance rate and distribution volume to the same extent. These results demonstrate that the absorption of multiple dosages of the drug was only 0.674 times that of a single dose. This phenomenon was probably induced by repeated subcutaneous injection, leading to the partitioning of the drug into local adipose tissue, thereby reducing drug absorption by the body. Other than the effect of dose number, we did not find that the other covariates could significantly affect the pharmacokinetic parameters of rhTNFR-Fc, probably due to the narrow population distribution of the subjects. It should be noted that the pharmacokinetics of rhTNFR-Fc in healthy Chinese subjects and in Chinese patients with AS were similar; 
we could not add this different covariate into any pharmacokinetic parameters to improve the final model. The stability and extrapolation of the model developed in the present study need further improvement and evaluation in a larger group of subjects before the model can be fully validated.

Interestingly, this analysis confirmed that the estimated population parameters in Chinese subjects were similar to those reported previously in other nations ${ }^{[20]}$. For example, CL/F was $0.110 \mathrm{~L} / \mathrm{h}$ for Chinese males and $0.168 \mathrm{~L} / \mathrm{h}$ for Chinese females, values similar to the CL/F values of $0.132 \pm 0.085 \mathrm{~L} / \mathrm{h}$ (mean $\pm \mathrm{SD}$ ) in American subjects. In addition, the mean population $\mathrm{V} / \mathrm{F}$ values observed in Chinese and American subjects were $15.5 \mathrm{~L}$ and $12 \mathrm{~L}$, respectively. However, it has been reported that non-Caucasian subjects have a greater clearance of rhTNFR-Fc than Caucasian subjects ${ }^{[18]}$, but that finding did not reach statistical significance due to an unbalanced design. Therefore, we cannot report that non-Caucasian individuals have a larger clearance of rhTNFR-Fc than Caucasians.

In conclusion, the population pharmacokinetics of rhTNFR$F_{C}$ in healthy Chinese volunteers and in Chinese patients with AS exhibited first-order kinetic characteristics that were best described by a one-compartment model. The pharmacokinetics of rhTNFR-Fc in healthy Chinese subjects and in Chinese patients with AS were similar. However, we found that Chinese females have a higher $\mathrm{CL} / \mathrm{F}$ than Chinese males and that multiple dosages can significantly decrease the absorption of rhTNFR-Fc. The PK parameters in Chinese subjects were similar to those reported for American subjects.

\section{Acknowledgements}

This work was financially supported by Celgen Bio-Pharmaceutical Co Ltd (Shanghai, China). The authors would like to thank Prof Rui WANG for direction of the clinical work. We are also thankful to Prof Feng HUANG, Prof Ze-ling CAI, Mr Xiao-hu DENG, and Ms Chun-hua YANG from General Hospital of PLA for their technical assistance. Thanks to our families for their understanding and support in our study, work, and life.

\section{Conflict of interest}

The authors declare that this work have no conflict of interest.

\section{Author contribution}

Wang R, Tang ZM and Guan HS designed research; Fang Y, Huang F, Song HF, and Li YZ performed research; Tang ZM contributed new analytical tools and reagents; Li LJ and Zheng QS analyzed data; Zheng QS and Li LJ wrote the paper.

\section{References}

$1 \mathrm{Ng}$ SC, Liao Z, Yu DT, Chan ES, Zhao L, Gu J. Epidemiology of spondyloarthritis in the People's Republic of China: review of the literature and commentary. Semin Arthritis Rheum 2007; 37: 39-47.

2 Gran JT, Skomsvoll JF. The outcome of ankylosing spondylitis: a study of 100 patients. Br J Rheumatol 1997; 36: 766-71.

3 Khalessi AA, Oh BC, Wang MY. Medical management of ankylosing spondylitis. Neurosurg Focus 2008; 24: E4.

4 Kalden JR. Emerging role of anti-tumor necrosis factor therapy in rheumatic diseases. Arthritis Res 2002; 4: S34-40.

5 Camussi G, Albano E, Tetta C, Bussolino F. The molecular action of tumor necrosis factor-alpha. Eur J Biochem 1991; 202: 3-14.

6 Crew MD, Effros RB, Walford RL, Zeller E, Cheroutre H, Brahn E. Transgenic mice expressing a truncated Peromyscus leucopus TNFalpha gene manifest an arthritis resembling ankylosing spondylitis. J Interferon Cytokine Res 1998; 18: 219-25.

7 Bal A, Unlu E, Bahar G, Aydog E, Eksioglu E, Yorgancioglu R. Comparison of serum IL-1 beta, sIL-2R, IL-6, and TNF-alpha levels with disease activity parameters in ankylosing spondylitis. Clin Rheumatol 2007; 26: 211-5.

8 Braun J, Bollow M, Neure L, Seipelt E, Seyrekbasan F, Herbst H, et al. Use of immunohistologic and in situ hybridization techniques in the examination of sacroiliac joint biopsy specimens from patients with ankylosing spondylitis. Arthritis Rheum 1995; 38: 499-505.

9 Francois RJ, Neure L, Sieper J, Braun J. Immunohistological examination of open sacroiliac biopsies of patients with ankylosing spondylitis: detection of tumour necrosis factor alpha in two patients with early disease and transforming growth factor beta in three more advanced cases. Ann Rheum Dis 2006; 65: 713-20.

10 Amgen-Wyeth Enbrel Prescribing Information. United States 2009.

11 Braun J, Sieper J, Breban M, Collantes-Estevez E, Davis J, Inman $\mathrm{R}$, et al. Anti-tumour necrosis factor alpha therapy for ankylosing spondylitis: international experience. Ann Rheum Dis 2002; 61: iii51-60.

12 Brandt J, Khariouzov A, Listing J, Haibel H, Sorensen H Grassnickel L, et al. Six-month results of a double-blind, placebo-controlled trial of etanercept treatment in patients with active ankylosing spondylitis. Arthritis Rheum 2003; 48: 1667-75.

13 Davis JC, Van Der Heijde D, Braun J, Dougados M, Cush J, Clegg DO, et al. Recombinant human tumor necrosis factor receptor (etanercept) for treating ankylosing spondylitis: a randomized, controlled trial. Arthritis Rheum 2003; 48: 3230-6.

14 Baraliakos X, Davis J, Tsuji W, Braun J. Magnetic resonance imaging examinations of the spine in patients with ankylosing spondylitis before and after therapy with the tumor necrosis factor alpha receptor fusion protein etanercept. Arthritis Rheum 2005; 52: 1216-23.

15 Brandt J, Listing J, Haibel H, Sorensen H, Schwebig A, Rudwaleit M, et al. Long-term efficacy and safety of etanercept after readministration in patients with active ankylosing spondylitis. Rheumatology (Oxford) 2005; 44: 342-8.

16 Korth-Bradley JM, Rubin AS, Hanna RK, Simcoe DK, Lebsack ME. The pharmacokinetics of etanercept in healthy volunteers. Ann Pharmacother 2000; 34: 161-4.

17 Zhou H. Clinical pharmacokinetics of etanercept: a fully humanized soluble recombinant tumor necrosis factor receptor fusion protein. J Clin Pharmacol 2005; 45: 490-7.

18 Lee H, Kimko HC, Rogge M, Wang D, Nestorov I, Peck CC. Population pharmacokinetic and pharmacodynamic modeling of etanercept using logistic regression analysis. Clin Pharmacol Ther 2003; 73: 348-65.

19 Yim DS, Zhou H, Buckwalter M, Nestorov I, Peck CC, Lee H. Population pharmacokinetic analysis and simulation of the time-concentration profile of etanercept in pediatric patients with juvenile rheumatoid arthritis. J Clin Pharmacol 2005; 45: 246-56.

20 Kawai S, Sekino H, Yamashita N, Tsuchiwata S, Liu H, Korth-Bradley $\mathrm{JM}$. The comparability of etanercept pharmacokinetics in healthy Japanese and American subjects. J Clin Pharmacol 2006; 46: 41823. 